\title{
Thermodynamique des systèmes micellaires : Partie II - Volumes molaires apparent et partiel des alcanoates de sodium selon la méthode de la pseudo-phase (MPP)
}

\author{
S. M. Haiji* et M. W. O. Sellahi \\ Laboratoire de chimie organique et d'études physico-chimiques, E.N.S. Takaddoum, \\ avenue Oued Akreuch, B.P. 5118, Rabat, Maroc \\ (Reçu le 16 Février 2006, accepté le 25 Juillet 2006)

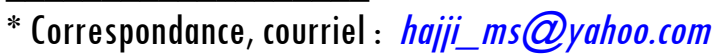

\section{Résumé}

Les masses volumiques des différentes solutions de la série homologue des alcanoates de sodium du formiate $(\mathrm{n}=0)$ à l'undécanoate $(\mathrm{n}=10)$ ont été étudiées selon le modèle de la pseudo-phase (MPP). Ce modèle nous a permis d'introduire deux concentrations critiques délimitant la zone de Ila pseudo-phasell. Nous avons déterminé d'une part des domaines de Imicro-phasell qui sont des domaines d'équilibre où les micelles peuvent exister, et d'autre part des domaines de "micro- hétérogénéitéll où la structure serait instable et variable avec la concentration. De même, on a pu déterminer les volumes molaires apparent et partiel de chacun des produits ainsi que la variation du volume durant la micellisation. La méthode a permis d'accéder à un certain nombre de grandeurs micellaires.

Mots-clés : densimétrie, volumes molaires apparent et partiel, micelle, interactions soluté-solvant et soluté-soluté, alcanoate de sodium.

\section{Abstract}

Thermodynamics of micellar systems : Part II - Apparent and partial molar volumes of sodium alkanoates according to the pseudo-phase model (MPP)

Volumetric measurements were carried out to obtain the apparent and partial molar volume of the aqueous solutions of the homologous series of sodium alkanoates from formate $(n=0)$ to undecanoate $(n=10)$. The results are analysed according to the pseudophase method (MPP). Three areas are defined corresponding to "the authentic solutions, 
micro-heterogeneity and pseudo-phase". Then the critical micellar concentrations of products were obtained and the variations of the apparent and partial molar volumes during the micellization were determined. This method permits also to evaluate different micellar properties.

Keywords : densimetry, apparent and partial molar volumes, micelle, solute-solvent and solute-solute interactions, sodium alcanoate.

\section{Introduction}

Les études thermodynamiques concernant les solutions aqueuses des alcanoates de sodium sont nombreuses et très diverses [1-5]. Les propriétés volumiques des alcanoates de sodium ont été largement étudiées en particulier par Haiii [6], Sakurai [7], Redlich [8], King [9], Vikingstad [10], et Douheret [11]. D'après ces différents travaux, les auteurs ont conclu que l'acétate et le formiate de sodium ne présentent pas d'associations et que les homologues supérieurs forment des agrégats à partir du propanoate. Dans une communication récente [12 ], nous avons étudié cette même série des alcanoates de sodium par la méthode d'action de masse et nous avons montré l'existence des concentrations micellaires critiques à partir de l'hexanoate de sodium. Des études concernant les capacités calorifiques molaires apparentes portant sur l'acétate et l'octanoate de sodium ont été réalisées par Leduc [13], alors que le décanoate de sodium a été étudié par Delisi [4].

La distinction entre les phénomènes associatifs et micellaires, souvent n'étant pas très aisée, notre but est d'apporter une contribution à la compréhension des phénomènes de formation de structure micellaire, d'agrégations et d'associations progressives attribuées généralement à des interactions hydrophobes [14,15].

Nous présentons ici les résultats d'une étude par volumétrie des solutions aqueuses d'alcanoates de sodium $\mathrm{H}-\left(\mathrm{CH}_{2}\right)_{n}-\mathrm{COO}^{-} \mathrm{Na}^{+}$avec $\mathrm{n}=0$ à 10 . Cette technique a été utilisée pour la détermination des concentrations micellaires critiques (cmc) et des volumes molaires apparents et partiels. Ensuite nous discutons les interactions soluté-solvant et soluté-soluté.

\section{Matériel et méthodes}

\section{2-1. Rappels}

De nombreux auteurs ont utilisé, pour le traitement des données expérimentales le modèle de la peudo-phase $[11,16]$. Nous donnons ici un bref rappel: 
Le modèle de la pseudo-phase proposé et développé par Shinoda [17] a été appliqué par d'autres auteurs $[5,10,11,16]$ pour l'explication de la formation de la micelle. Ce modèle traite la micelle d'une manière analogue à une phase séparée et suppose un certain nombre d'approximations à savoir : association en une seule étape - coefficient d'activité constant au-dessus de la $\mathrm{cmc}$ - La cmc est considérée comme une concentration de saturation [18] qui, si elle est dépassée, donne lieu à une phase nouvelle - enfin la micellisation engendre un nombre d'agrégation unique, ce qui n'est pas le cas dans les vraies phases de séparation [17].

\section{2-2. Produits et techniques expérimentales}

\section{2-2-1. Produits}

Les alcanoates de sodium utilisés ont été obtenus par neutralisation de l'acide correspondant. Ces acides, d'origines différentes, ont été distillés sous pression réduite immédiatement avant neutralisation par une solution titrée normale de soude. Les sels obtenus, après évaporation de l'eau au bain-marie, ont été séchés à l'étuve à $110^{\circ} \mathrm{C}$ pendant 48 heures, puis au dessiccateur (sur anhydride phosphorique) pendant trois jours jusqu'à élimination totale de l'eau.

Le formiate et l'acétate de sodium (ultra pur Normaton) dont la teneur maximale en impureté est de $10^{-4} \%$, furent utilisés sans purification supplémentaire. Le propionate de sodium pur à $99 \%$ a été filtré à chaud, recristallisé puis lavé à l'éthanol absolu à froid. Ces trois derniers sels ont été séchés à l'étuve puis au dessiccateur comme vu précédemment.

\section{2-2-2. Techniques expérimentales}

Les densités des solutions sont déterminées à $298,15 \mathrm{~K}$ par un densimètre commercial version SODEV (02D) [19]. Son principe a été détaillé dans une étude antérieure [12]. La masse volumique de la solution est donnée par la relation suivante :

$$
\rho=A+K(\tau)^{2}
$$

$A$ et $K$ sont des constantes de calibrage du système à une température donnée. $\tau$ est la période correspondant à la fréquence de résonance du tuyau oscillateur. Les fluctuations de la température doivent être maintenues en deçà de $10^{-3}{ }^{\circ} \mathrm{C}$, car la masse volumique de la plupart des liquides varie de quelques centaines de ppm par degré $\left(e l s i u s ~\left({ }^{\circ} \mathrm{C}\right)\right.$. Pour des mesures différentielles, la relation (l) se réduit à :

$$
\rho^{t^{0}}=\rho_{0}^{t^{0}}+K t^{0}\left(\tau^{2}-\tau_{0}^{2}\right)
$$

\section{S. M. Haiji et M. W. O. Sellahi}


où $\rho_{0}$ et $\tau_{0}$ se réfèrent au liquide de référence (généralement le solvant pur) et $t$ la température à laquelle on travaille.

Avec cette technique, les différences sur les masses volumiques peuvent être mesurées à $\pm 3 \times 10^{-6} \mathrm{~g} . \mathrm{cm}^{-3}$ près, avec environ $5 \mathrm{~cm}^{3}$ du liquide pour une durée de $5 \mathrm{mn}$. Cette incertitude correspond approximativement à $\pm 10^{-4}$ mole. $\mathrm{kg}^{-1}$ sur la concentration des électrolytes simples dans l'eau. Cette technique est très sensible aux modifications de structure qui interviennent dans la solution. Elle a l'avantage de ne pas modifier l'équilibre des espèces en solution par l'introduction de substances nouvelles.

A partir des masses volumiques, on calcule les différentes grandeurs molaires tels que le volume molaire apparent $\Phi_{v}$ ef le volume molaire partiel $V_{2}$ via les relations suivantes:

$$
\begin{aligned}
& \Phi_{v}=\frac{M}{\rho}-1000\left[\frac{\rho-\rho_{0}}{\rho \rho_{0} m}\right] \\
& \bar{V}_{2}=\Phi_{v}+m\left[\frac{\partial \Phi_{v}}{\partial m}\right]
\end{aligned}
$$

où $M$ désigne la masse molaire du corps dissout, $m$ la molalité de la solution, $\rho$ sa masse volumique et $\rho_{0}$ la masse volumique du solvant pur.

$V_{2}$ est déduit directement de $\Phi_{v}[16,20,21]$. Son extrapolation à dilution infinie ainsi que celle de $\Phi_{v}$ permet d'accéder au volume molaire apparent à dilution infinie $\left(V_{2}=\Phi_{v}^{0}\right)$. Pour les produits ioniques, selon la relation de Redlich-Meyer [22] donnée pour les électrolytes $1: 1$, on a dans la région pré-micellaire :

$$
\Phi_{v}-A_{v}\left(\rho_{0} m\right)^{1 / 2}=\Phi_{v}^{0}+B_{v} m
$$

où $A_{v}$ est la pente limite de la loi Debye-Huckel (à $25^{\circ} \mathrm{C}$, $A_{v}$ est égale à $1.686 \mathrm{~cm}^{3 / 2} \cdot \mathrm{mol}^{-3 / 2}$ [23], $\left.1.865 \mathrm{~cm}^{3 / 2} \cdot \mathrm{mol}^{-3 / 2}[24]\right)$, et $B_{v}$ un paramètre ajustable qui mesure la déviation à cette loi. Les solutions sont préparées par pesée avec une balance sensible à $5.10^{-5} \mathrm{~g}$. L'eau utilisée est déminéralisée, bidistillée puis filtrée sur des filtres millipore de $0.45 \mu \mathrm{m}$. 


\section{Résultats et discussion}

\section{3-1. Modèle de la pseudo-phase (M.P.P.)}

Dans cette approche, une propriété extensive p est définit par :

$$
\left(\Phi_{p}\right)_{2}=\left(\frac{p-n_{1} p_{1}^{0}}{n_{2}}\right)
$$

où $n_{1}$ et $n_{2}$ sont les nombres de moles de solvant et de soluté, $p_{1}{ }^{0}$ est la grandeur molaire pour le corps pur $i$. Si $\mathrm{p}^{\mathrm{E}}$ et $\mathrm{x}_{2}$ sont les grandeurs molaires d'excès et la fraction molaire du soluté, on a alors:

$$
\left(\Phi_{p}\right)_{2}=p_{2}^{0}+\frac{p^{\mathrm{E}}}{\mathrm{X}_{2}}
$$

Or, on montre qu'en milieu diphasique, $\mathrm{p}^{\mathrm{E}}$ est sous la forme :

$$
p^{\mathrm{E}}=\boldsymbol{a} \mathrm{X}_{2}+\boldsymbol{b}
$$

soit pour

$$
\left(\Phi_{p}\right)_{2}=a^{\prime}+\frac{b^{\prime}}{\mathrm{X}_{2}}
$$

De ce fait, un milieu diphasique sera donc caractérisé par une évolution linéaire de la grandeur molaire apparente en fonction de l'inverse de la fraction molaire (ou de la molalité) du soluté. Si, dans un certain domaine, pour une grandeur extensive, une telle loi est vérifiée, le système pourra être traité comme un milieu diphasique.

La règle des phases impose pour un système binaire une invariance des propriétés des phases en équilibre. Un tel modèle implique une invariance de structure de ces pseudophases. Nous parlerons dans ce cas, de micro-phase par opposition aux domaines de micro-hétérogénéité dont la structure sera variable avec la concentration.

Ainsi, la concentration micellaire critique sera considérée comme une micro-phase homogène [25] où une seule entité existe. Au-dessus de cette concentration, une autre espèce de taille, de forme et de structure peut se former, donnant lieu à une deuxième micro-phase. Selon Hartley [26], l'accroissement de la concentration conduit seulement à une modification de la géométrie de la micelle.

L'explication thermodynamique en terme de changement de volumes molaires apparent et partiel a été réalisée par Desnoyers [16]. II conclue que la différence des volumes molaires partiels entre les phases au-dessus (b) et au-dessous (a) de la cmc sera : 


$$
\bar{V}_{2}{ }^{b}-\bar{V}_{2}{ }^{0}=\Delta \bar{V}_{m}+(m-c m c) \frac{\delta\left(\Delta \bar{V}_{m}\right)}{\delta m}
$$

ò̀ $\Delta \bar{V}_{m}$ est la variation du volume molaire pendant la phase de micellisation calculée à partir des valeurs correspondant aux points singuliers au-dessus (b) et au-dessous (a) de la cmc, m étant la molalité de la solution.

A la cmc, le deuxième terme de cette relation s'annule et l'équation (10)devient :

$$
\bar{V}_{2}^{b}-\bar{V}_{2}^{0}=\Delta \bar{V}_{m}
$$

Zana [5] et Vikingstad [10] ont utilisé la même méthode pour calculer les volumes molaires apparents.

\section{3-1-1. Concentrations critiques distinctes (cc) délimitant différentes zones d'évolution du milieu}

Ce modèle va nous permettre d'introduire deux concentrations critiques délimitant la zone de pseudo-phase qui est une invariance de la structure des micro-phases. Nous aurons d'une part des domaines de micro-phase, qui seront des domaines d'équilibre où les micelles peuvent exister, par opposition aux domaines de micro-hétérogénéité où la structure serait instable et variable avec la concentration. De même, on pourrait accéder graphiquement aux propriétés thermodynamiques des phases en équilibre comme pour les milieux diphasiques authentiques. Si l'on désigne par $\alpha$ la phase aqueuse des solutions authentiques et par $\beta$ la micro-phase, on pourrait déterminer les valeurs des volumes molaires apparents des phases $\alpha$ et $\beta$ à savoir $\left(\Phi_{v}^{\alpha}\right)_{2}$ et $\left(\Phi_{v}^{\beta}\right)_{2}$.

La caractérisation du milieu diphasique par une variation linéaire de $\Phi_{v}$ en fonction de l'inverse de la molalité a été suggérée par Douheret [11]. Elle permet d'appliquer le modèle de la pseudo-phase à la série des alcanoates de sodium du formiate à l'undécanoate $(\mathrm{n}=0$ à 10$)$. Les courbes $\Phi_{v}=f(1 / \mathrm{m})$ évoluent avec la longueur de la chaîne hydrocarbonée. 0 n relève trois cas de figures :

- Pour $\mathrm{n}<2$, les courbes ne présentent aucune partie linéaire et aucun point critique n'est discernable. On peut tout au plus confondre la courbe avec sa tangente pour les plus faibles valeurs de $1 / \mathrm{m}$ (Figure 1 ).

- Pour $2 \leq \mathrm{n} \leq 5$, les courbes font apparaître une linéarité très réduite à partir d'une concentration critique $\left(m_{b}\right)$ dans un domaine étroit de forte concentration (Figure 2).

- Pour $6 \leq \mathrm{n} \leq 10$, en solution diluée, les courbes présentent une variation régulière correspondant aux zones des solutions authentiques limitées par la concentration $\left(\mathrm{m}_{\mathrm{a}}\right)$. 
Au fur et à mesure que la concentration augmente, il apparaît d'abord une zone de microhétérogénéité entre les concentrations $\left(m_{0}\right)$ et $\left(m_{b}\right)$, puis une zone de variation linéaire correspondant au domaine de la micro-phase délimitée par les concentrations $\left(m_{b}\right)$ et $\left(m_{c}\right)$ (Figure 3). Par ailleurs, l'undécanoate de sodium ( $\mathrm{n}=10)$, en zone très concentrée, présente un deuxième domaine de micro-hétérogénéité au-dessus de $\left(\mathrm{m}_{\mathrm{c}}\right)$.

On admet donc que seules les régions de la structure de la micro-phase demeurent inchangées. Ils leur correspondent des grandeurs volumiques invariantes responsables des domaines linéaires compris entre les concentrations $\left(m_{b}\right)$ et $\left(m_{c}\right)$. Nous avons consigné dans le Tableav $I$ les différents résultats correspondants à la série homologue des alcanoates de sodium. On y trouve respectivement:

- Les concentrations critiques $m_{a}$ correspondant au début de la formation des micelles (début du domaine de micro-hétérogénéité),

- Les concentrations critiques $m_{b}$ correspondant à la fois à la fin du domaine de microhétérogénéité et au début de celui de la micro-phase,

- La concentration critique $\mathrm{m}_{\mathrm{c}}$ correspondant à la fin du domaine de la micro-phase et au début de celui de l'évolution des micelles.

Tableau 1 : Propriétés volumiques des alcanoates de sodium calculées à $25^{\circ} \mathrm{C}$ selon le modèle de la séparation de phases

\begin{tabular}{|c|c|c|c|c|c|c|c|c|}
\hline $\mathrm{n}$ & $\begin{array}{l}\mathrm{ma} \\
\left(\mathrm{mol} . \mathrm{L}^{-1}\right)\end{array}$ & $\begin{array}{l}\mathrm{mb} \\
\left(\mathrm{mol} \mathrm{L}^{-1}\right)\end{array}$ & $\begin{array}{l}\mathrm{mc} \\
\left(\mathrm{mol} \mathrm{LL}^{-1}\right)\end{array}$ & $\begin{array}{l}\bar{V}_{2}^{\alpha}=\left(\Phi^{\alpha}{ }_{v}\right)_{2} \\
\left(\mathrm{~cm} 3 . \mathrm{mol}^{-1}\right)\end{array}$ & $\begin{array}{l}\left.\bar{V}_{2}^{\beta}=\left(\Phi^{\beta}\right)_{v}\right)_{2} \\
\left(\mathrm{~cm} 3 . \mathrm{mol}^{-1}\right)\end{array}$ & $\begin{array}{l}\left|\Delta \overline{\mathrm{V}_{\mathrm{m}}}\right|^{\beta} \alpha \\
\left(\mathrm{cm} 3 . \mathrm{mol}^{-1}\right)\end{array}$ & $\alpha$ & B \\
\hline 0 & 0,68 & - & - & - & - & - & - & - \\
\hline 1 & 0,5 & - & - & - & - & - & - & - \\
\hline 2 & 0,74 & - & - & - & - & - & - & - \\
\hline 3 & 0,32 & 2,45 & - & 70,20 & $\begin{array}{c}80,61 \\
81,50^{*}\end{array}$ & $\begin{array}{l}10,41 \\
12,1^{*}\end{array}$ & $-0,04$ & - \\
\hline 4 & 0.096 & 1.74 & - & 85.83 & $\begin{array}{l}96,38 \\
97,0^{*}\end{array}$ & $\begin{array}{l}10,55 \\
11,8^{*}\end{array}$ & $-0,006$ & - \\
\hline 5 & 0,15 & 1,56 & - & 101,47 & $\begin{array}{l}113,33 \\
112,5^{*}\end{array}$ & $\begin{array}{c}11,86 \\
11,10^{*}\end{array}$ & $-0,002$ & $\begin{array}{c}-15,07 \\
-12^{*}\end{array}$ \\
\hline 6 & 0,38 & 1,15 & - & 118,04 & $\begin{array}{c}129,1 \\
128,5^{*}\end{array}$ & $\begin{array}{c}11,06 \\
11,10^{*}\end{array}$ & $-0,046$ & $\begin{array}{c}-5,9 \\
-8,1^{*}\end{array}$ \\
\hline 7 & 0,29 & 0,54 & 1,03 & 133,4 & $\begin{array}{c}143,7 \\
143,5^{*} \\
\end{array}$ & $\begin{array}{c}10,3 \\
11,0^{*}\end{array}$ & $-0,07$ & $\begin{array}{l}-3,35 \\
-4,3^{*} \\
\end{array}$ \\
\hline 8 & 0,08 & 0,22 & 0,44 & 149,2 & $\begin{array}{c}159,2 \\
159,5^{*} \\
\end{array}$ & $\begin{array}{c}10,0 \\
11,00^{*}\end{array}$ & -0.023 & $\begin{array}{r}-2,57 \\
-2,32 * \\
\end{array}$ \\
\hline 9 & 0,041 & 0,31 & 0,62 & 165,5 & $\begin{array}{c}175,2 \\
175,5^{*} \\
\end{array}$ & $\begin{array}{c}9,7 \\
11,40^{*} \\
\end{array}$ & $-0,015$ & $\begin{array}{c}-1,75 \\
-1,23^{*} \\
\end{array}$ \\
\hline 10 & 0,032 & 0,09 & 0,59 & 180,9 & $\begin{array}{c}189.0 \\
191,3^{*}\end{array}$ & $\begin{array}{c}8,1 \\
11,40^{*}\end{array}$ & $-0,011$ & $\begin{array}{l}-0,56 \\
-0,7^{*}\end{array}$ \\
\hline
\end{tabular}

$* \operatorname{Ref}[11]$ 


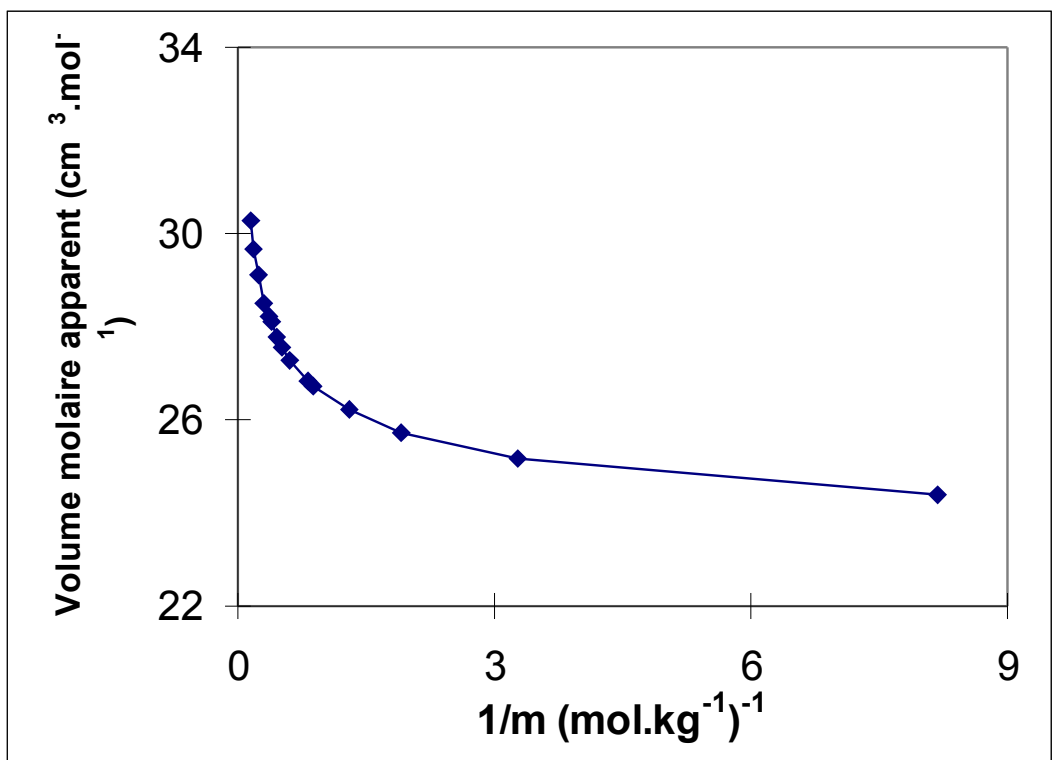

Figure 1 : Volume molaire apparent du formiate de sodium $(n=0)$ selon le modèle de la pseudo-phase (MPP)

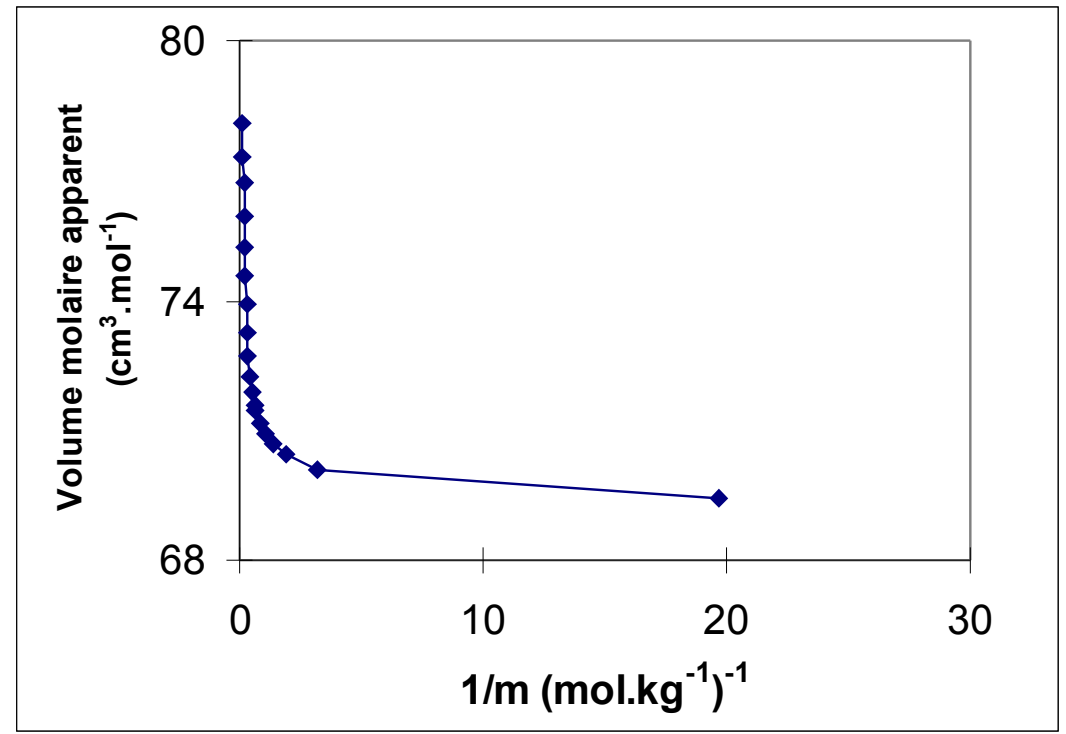

Figure 2 : Volume molaire apparent du butanoate de sodium $(n=3)$ selon le modèle de la pseudo-phase (MPP) 


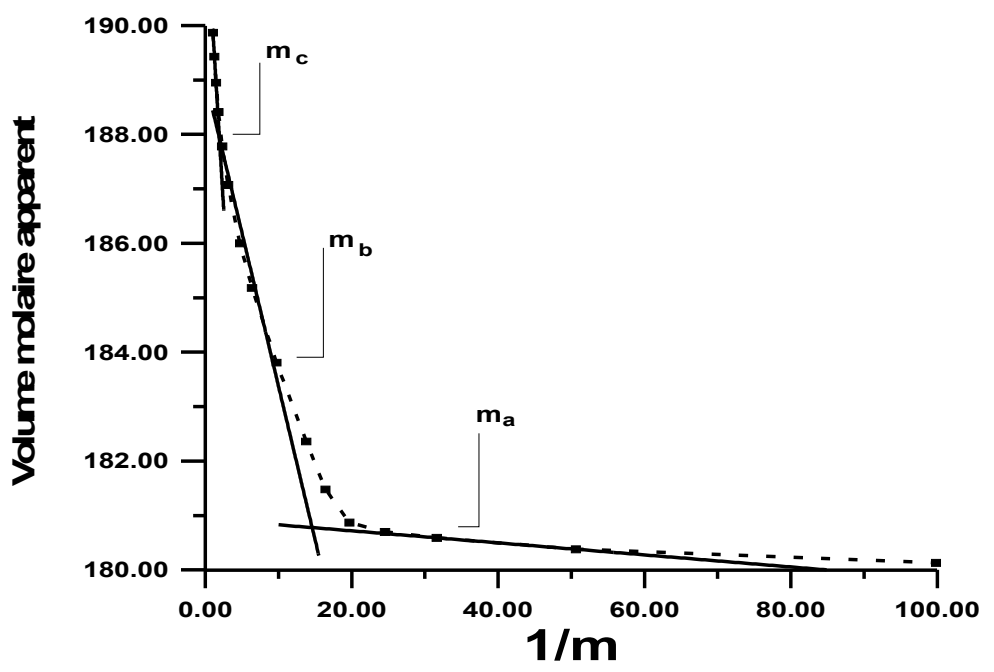

Figure 3 : Volume molaire apparent de l'undécanoate de sodium $(n=10)$ selon le modèle de la pseudo-phase (MPP)

Un traitement statistique des données nécessite un nombre élevé de points expérimentaux dans chaque région pour confirmer l'existence des domaines de la microphase et de la micro-hétérogénéité.

Une étude similaire réalisée par Douheret [11] sur l'octanoate de sodium à partir des résultats d'Ekwall [27] confirme aisément l'existence des différents domaines que nous avons définis ci-dessus.

\subsubsection{Grandeurs molaires}

Le traitement des propriétés extensives permet de caractériser les milieux diphasiques par des grandeurs proportionnelles à l'inverse de la fraction molaire ou de la molalité. Ainsi, le volume molaire apparent d'une pseudo-phase sera défini par :

$$
\left(\Phi_{v}\right)_{2}=\left(\Phi_{v}^{\beta}\right)_{2}+\beta m^{-1}
$$

où $\beta$ représente la pente de la droite et $\left(\Phi_{v}^{\beta}\right)_{2}$ le volume molaire apparent de la microphase $\beta$.

L'extrapolation de l'équation (12) à $m^{-l}=0$ permet d'atteindre les différentes valeurs des volumes molaires apparents.

Si l'on considère que la micro-phase est similaire à un corps pur, le volume molaire apparent du soluté dans ce domaine sera égal à son volume molaire partiel $\left(\Phi_{v}^{\beta}\right)_{2}=\bar{V}_{2}^{\beta}$ 
L'ensemble de ces valeurs est consigné dans le Tableau 1. On y trouve :

- Les volumes molaires apparents $\left(\Phi_{v}^{\alpha}\right)_{2}$ et les pentes $\alpha$ des solutions authentiques,

- Les volumes molaires apparents $\left(\Phi_{v}^{\beta}\right)_{2}$ et les pentes $\beta$ des premiers domaines microphasiques,

- Les variations des volumes molaires $\left|\Delta \bar{V}_{m}\right|_{\alpha}^{\beta}$ lors du passage des domaines des solutions authentiques aux premiers domaines pseudo-phasiques.

Les valeurs des $\left|\Delta \bar{V}_{m}\right|_{\alpha}^{\beta}=\bar{V}_{2}^{\beta}-\bar{V}_{2}^{\alpha}$ sont positives et augmentent légèrement en passant de $10,41 \mathrm{~cm}^{3} \cdot \mathrm{mol}^{-1}$ pour le butyrate $(\mathrm{n}=3)$ à $11,86 \mathrm{~cm}^{3} \cdot \mathrm{mol}^{-1}$ pour l'hexanoate $(\mathrm{n}=5)$, puis diminuent pour atteindre $8,1 \mathrm{~cm}^{3} \cdot \mathrm{mol}^{-1}$ pour l'undécanoate $(\mathrm{n}=10)$.

Un effet différent a été constaté [11], où $\left|\Delta \bar{V}_{m}\right|_{\alpha}^{\beta}$ décroît en allant du butyrate de $(\mathrm{n}=3)$ $\left(\left.\Delta \bar{V}_{m}\right|_{\alpha} ^{\beta}=12,15 \mathrm{~cm}^{3} \cdot \mathrm{mol}^{\prime}\right)$ à l'octanoate de sodium $\left(\left.\Delta \bar{V}_{m}\right|_{\alpha} ^{\beta}=11,0 \mathrm{~cm}^{3} \cdot \mathrm{mol}^{l}\right)$, puis croît à $11,40 \mathrm{~cm}^{3} \cdot \mathrm{mol}^{-1}$ pour le décanoate et l'undécanoate $(\mathrm{n}=9$ ef 10$)$.

Nous avons montré [20] lors de l'étude des volumes molaires apparents des bromures de $\mathrm{R}(-)$ (N,N-diméthyl, $\mathrm{N}$-alkyl $\left(\mathrm{C}_{n} \mathrm{H}_{2 n+1}\right)$ ammonio)-2 butanol par la méthode de la pseudophase que $\left|\Delta \bar{V}_{m}\right|_{\alpha}^{\beta}$ décrô̂t légèrement de $7,94 \mathrm{~cm}^{3} \cdot \mathrm{mol}^{-1}$ pour le décyle à $6,31 \mathrm{~cm}^{3} \cdot \mathrm{mol}^{-1}$ pour le dodécyle, puis elle remonte à $7,60 \mathrm{~cm}^{3} \cdot \mathrm{mol}^{-1}$ pour le tridécyle.

Jolicoeur [28] a constaté dans la série des détergents ioniques que la variation du volume croît avec la longueur de la chaîne et demeure néanmoins indépendante du contre ion.

\section{3-2. Dimensions micellaires}

De nombreux auteurs ont montré que les micelles ioniques possèdent en solution aqueuse une forme sphérique [29-32] qui se transforme en forme ellipsoïdale puis cylindrique à concentration plus élevée.

D'autres auteurs [33] ont attribué une forme d'ellipsoïde plate à des micelles de diverses amphiphiles.

Les conséquences thermodynamiques développées précédemment conduisent à considérer que dans le domaine de la micro-phase et dans le cas des micelles sphériques, celles-ci doivent avoir un rayon $R$ constant. La micro-phase inclut nécessairement l'ensemble des contre-ions, sinon l'addition de soluté entraînerait une

\section{S. M. Haiji et M. W. O. Sellahi}


variation de composition de la phase continue, ce qui serait contraire à la règle des phases. On aura :

$$
R=\frac{\left[3<N_{\beta}>\left(\Phi_{v}^{\beta}\right)_{2}\right]^{\frac{3}{2}}}{\left[4 T T N_{A}\right]^{\frac{3}{2}}}
$$

et connaissant $R$, on peut déduire la surface libre moyenne par tête polaire à l'interface via la relation suivante :

$$
<S>=\frac{4 \Pi R^{2}}{<N_{\beta}>}
$$

où $N_{A}$ est le nombre d'Avogadro, $\left\langle N_{\beta}>\right.$ le nombre moyen d'agrégations et $\left(\Phi_{v}^{\beta}\right)_{2}$ le volume molaire partiel de la pseudo-phase.

Tableau 2 : Dimensions micellaires des alcanoates de sodium calculées selon un modèle sphérique appliqué à la micro-phase $\beta$

\begin{tabular}{cccccc}
\hline $\mathbf{n}$ & $<\mathbf{N}_{\beta}>$ & $\mathbf{R}(\AA)$ & $\mathbf{R}(\AA)$ & $\left\langle\mathbf{S}>\left(\AA^{2}\right)\right.$ & $\left\langle\mathbf{S}>\left(\AA^{2}\right)\left(^{* *}\right)\right.$ \\
\hline 3 & $8^{*}$ & 6,35 & $6,37^{*} ; 6,39^{* *}$ & 63.31 & 63,7 \\
4 & $11^{*}$ & 7,50 & $7,51^{*} ; 7,66^{* *}$ & 64.23 & 64,4 \\
5 & $15^{*}$ & 8,76 & $8,75^{*} ; 8,93^{* *}$ & 64.25 & 64,1 \\
6 & $20^{*}$ & 10,07 & $10,06^{*} ; 10,20^{* *}$ & 63.68 & 63,6 \\
7 & $25^{*}$ & 11,20 & $11,25^{*} ; 11,47^{* *}$ & 63.02 & 63,6 \\
8 & $31^{*}$ & 12,44 & $12,51^{*} ; 12,74^{* *}$ & 62.70 & 63,5 \\
9 & $39^{*}$ & 13,91 & $13,95^{*} ; 14,01^{* *}$ & 62.31 & 62,7 \\
10 & $48^{*}$ & 15,27 & $15,38^{*} ; 15,28^{* *}$ & 61.01 & 62,0 \\
& & & & &
\end{tabular}

Dans le Tableau 2, nous avons rassemblé les valeurs trouvées pour la série des alcanoates de sodium. Nous remarquons que les rayons calculés sont en bon accord avec ceux trouvés par Douheret [11] d'une part, et avec ceux calculés par la méthode de Pauling [34] d'autre part. Une valeur moyenne de $63.09 \AA^{2}$ correspondant à la surface libre moyenne par tête polaire à l'interface a été trouvée. 


\section{Conclusion}

Grâce aux traitements appropriés des résultats par la méthode de la pseudo- phase, il a été possible de montrer, avec plus de précision, les zones de changement survenu au niveau structural dans de larges domaines de concentrations étudiées pour chaque amphiphile. Ces changements nous ont conduit à définir thermodynamiquement les concentrations qui correspondent aux domaines des solutions authentiques, des microhétérogénéités et des micro-phases. Nous avons pu aussi déterminer les quantités volumiques apparentes et partielles correspondant aux différents domaines.

Le modèle de la pseudo-phase a permis de montrer l'existence des interactions hydrophobes croissantes avec l'allongement de la chaîne hydrocarbonées conduisant à la formation des micelles pour les produits à $\mathrm{n} \leq 6$.

Ainsi l'existence d'une micro-phase au sens défini plus haut est à exclure pour le formiate et l'acétate de sodium $(n<2)$, pour lesquels, seule une phase de dispersion du tensioactif en milieu aqueux est à considérer. Alors que le propanoate, le butanoate, le pentanoate et l'hexanoate de sodium $(2 \leq \mathrm{n} \leq 5)$ présentent une structure prémicellaire stabilisée à concentration élevée. Seuls l'heptanoate, l'octanoate, le nanoate, le décanoate et l'undécanoate de sodium $(6 \leq n \leq 10)$ présentent une micro-phase au sens pré-défini plus haut et forment de vraies micelles.

\section{Remerciements}

Nous remercions Monsieur le professeur R. R. Durand de l'Université de Paris 13, pour sa disponibilité et pour les fructueuses discussions concernant ce travail.

\section{Références}

[1] - C. E. Larrabee and E.D. Sprague, J. Colloid and Interf. Sci., 114(1) (1986) 256-260.

[2] - J. B. Rosenholm, Ph.D. Dissertation, Abo Akademi, Finland, (1978).

[3] - R. Friman, K. Pettersson et P. Stenius, J. Colloid and Interf. Sci., 53(1) (1975) 90-101.

[4] - R. Delisi, G. Perron, J.E. Desnoyers, Can. J. Chem., 58 (1980) 959.

[5] - K. M. Kale, R. Zana, J. Colloid and Interf. Sci, 61(2) (1977) 313-322.

[6] - S.M. Haiji, M. Moussa et M.W.O. Sellahi, Premières Journées Nationales sur les Micelles, les microémulsions et les Tensioactifs, Settat, Maroc, 11 Mars 1999.

[7] - M. Sakurai and coll., Bulletin Chem. Soc. Jap., 48 (1975) 3494-3495.

[8] - Redlich and Nielson, J. Amer. Chem. Soc., 64 (1942) 761-762.

[9] - E. J. King, J. Phys.Chem., 73 (1969) 1220-1232.

[10] - E. Vikingstad, A. Skauge and H. Hailand, J. Colloid and Interf. Sci., 66(2) (1978) 240-246. 
[11] - G. Douheret et A. Viallard, J. de Chimie Physique, 78(1) (1981) 85-98.

[12] - S. M. Haiji et M. W. O. Sellahi, Afrique Science, Vol.1, N² (2005) 277-294, http://www.afriquescience.org

[13] - P. A. Leduc and J.E. Desnoyers, Can. J. Chem., 51 (1973) 2993-2998.

[14] - P. Mukeriee, Ber., Bunsen Phys. Chem., 82 (1978) 931-937.

[15] - W. Hess et R. Klein, J. Phys. A. : Math. Gen. 13, (1980) L5.

[16] - J. E. Desnoyers, R. Delisi, G. Perron, Pure \& Apl. Chem., 52 (1980) 433-444.

[17] - K. Shinoda and E. Hutchinson, J. Phys. Chem., 66 (1962) 577-582.

[18] - E. Hutchinson, A. Inaba, and L.G. Badey, Z. Physik. Chem. (Frankfurf), 5 (1955) 344.

[19] - E. W. Anacker and A. L. Underwood, J. Phys. Chem., 85 (1981) 2463-2466.

[20] - S. M. Haiji, Thèse de doctorat d'état ès sciences physiques, Univ. Paris Nord, (1987).

[21] - Landolt-Bornstein, New-Series IV/1b "Densities of binary aqueuse systems and heat capacities of liquid systems" Springer-verlag, Berlin-Heidelberg-New-york, (1977) 1.

[22] - 0. Redlich and D. M. Meyer, Chem. Rev., 64 (1964) 221-227.

[23] - L. A. Dunn, Trans. Faraday Soc., 64 (1968) 2951.

[24] - R. G. Bates, "Dermination of pH", Wiley, N. Y. (1964) 406.

[25] - K. Shinoda, T. Nakagawa, B. Tamamushi and T. Isemura, "colloid Surfactant" Some Physico-chemical Proprieties, Academic Press, New York, (1963) p 25-42.

[26] - G. S. Harteley "Micellization, Solubilisation and Microemulsion", Edité par K. L. Mittal, Plenum Press, New York, (1977) p. 23.

[27] - P. Ekwall, H. Eikrem and L. mandell, Acta. Chem. Scand., 17(1) (1963) 111-122.

[28] - C. Jolicoeur and G. Lacroix, Can. J. Chem., 54 (1976) 624.

[29] - N. A. Campbell, E M Kartzmark et G. R. Lakshminarayanan, Can. J. Chem., 40 (1962) 831.

[30] - P. J. Missel, N. A. Mazer, G. B. Benedekand and M. C. Capey, J. Phys. Chem., 87 (1983) 1264-1277.

[31] - M. Drifford, L. Belloni and M. Dubois, J. of Colloid and Interf. Sci., 118 (1) (1987) 50-67.

[32] - J. W. Mac Bain et A. Hoffmann, J. Chem. Phys., 16 (1948) 156.

[33] - H. V. Tartar, J. Phys. Chem., 59 (1955) 1195.

[34] - L. Pauling, The Nature of the Chemical Bound, Corne, Univ., Press, New York, (1960). 